Groups Geom. Dyn. 5 (2011), 553-565

DOI $10.4171 / \mathrm{GGD} / 139$
Groups, Geometry, and Dynamics

(C) European Mathematical Society

\title{
Reduction theory of point clusters in projective space
}

\author{
Michael Stoll
}

Dedicated to the memory of Fritz Grunewald

\begin{abstract}
We generalise earlier results of John Cremona and the author on the reduction theory of binary forms, whose zeros give point clusters in $\mathbb{P}^{1}$, to point clusters in projective spaces $\mathbb{P}^{n}$ of arbitrary dimension. In particular, we show how to find a reduced representative in the $\operatorname{SL}(n+1, \mathbb{Z})$-orbit of a given cluster. As an application, we show how one can find a unimodular transformation that produces a small equation for a given smooth plane curve.
\end{abstract}

Mathematics Subject Classification (2010). 11E10, 11H46, $11 \mathrm{E} 39$.

Keywords. Reduction theory, point clusters.

\section{Introduction}

In this paper, we generalise the results of [8] on the reduction theory of binary forms, which describe positive zero-cycles in $\mathbb{P}^{1}$, to positive zero-cycles (or point clusters) in projective spaces of arbitrary dimension. This should have applications to more general projective varieties in $\mathbb{P}^{n}$, by associating a suitable positive zero-cycle to them in an PGL $(n+1)$-invariant way. We discuss this in the case of (smooth) plane curves.

The basic problem motivating this work is as follows. Consider projective varieties over $\mathbb{Q}$ in some $\mathbb{P}^{n}$, with fixed discrete invariants. On this set, there is an action of $\operatorname{SL}(n+1, \mathbb{Z})$ by linear substitution of the coordinates. We would like to be able to select a specific representative of each orbit, which we will call reduced, in a way that is as canonical as possible. Hopefully, this representative will then also allow a description as the zero set of polynomials with fairly small integer coefficients.

Recall the main ingredients of the approach taken in [8]. The key role is played by a map $z$ from binary forms of degree $d$ into the symmetric space of $\operatorname{SL}(2, \mathbb{R})$ (which is the hyperbolic plane $\mathcal{H}$ in this case) that is equivariant with respect to the action of $\operatorname{SL}(2, \mathbb{Z})$. We then define a form $F$ to be reduced if $z(F)$ is in the standard fundamental domain for $\operatorname{SL}(2, \mathbb{Z})$ in $\mathscr{H}$. In order to make the map $z$ as canonical as possible, we use a larger group than $\operatorname{SL}(2, \mathbb{Z})$, namely $\operatorname{SL}(2, \mathbb{C})$; we then look for 
a map $z$ from binary forms with complex coefficients into the symmetric space $\mathscr{H}_{\mathbb{C}}$ for $\operatorname{SL}(2, \mathbb{C})$ that is $\operatorname{SL}(2, \mathbb{C})$-equivariant and commutes with complex conjugation. This map restricted to real forms will have image contained in $\mathscr{H}$ and satisfy our initial requirement.

Now there are in general many possible such maps $z$ (for exceptions, see Remark 12 below). We therefore need to pick one of them. In [8] this is achieved by a geometric property: we define a function on $\mathscr{H}_{\mathbb{C}}$, depending on $F$, that measures how far a point is from the roots of $F$ (up to an arbitrary additive constant); the covariant $z(F)$ is then the unique point in $\mathscr{H}_{\mathbb{C}}$ minimising this distance. This is essentially the same approach (but in a different interpretation) as that used by Julia in his thesis [5], who works out what $z(F)$ is for $F$ of degree 3 or 4 , but defines it more generally. $\mathrm{He}$ did not prove that his covariant is always well-defined, though. Julia was building on previous work by Hermite [3], [4]. For a more detailed discussion, see [8].

In our more general situation, we work with the space $\mathscr{H}_{n, \mathbb{R}}$ of positive definite quadratic forms in $n+1$ variables, modulo scaling, and the space $\mathscr{H}_{n, \mathbb{C}}$ of positive definite Hermitian forms in $n+1$ variables, modulo scaling (by positive real factors). There is a natural action of complex conjugation on $\mathscr{H}_{n, \mathbb{C}} ;$ the subset fixed by it can be identified with $\mathscr{H}_{n, \mathbb{R}}$.

We use the formula for the distance function mentioned above to obtain a similar function on $\mathscr{H}_{n, \mathbb{C}}$, depending on a collection of points in $\mathbb{P}^{n}(\mathbb{C})$. Under a suitable condition on the point cluster or zero-cycle $Z$, this distance function has a unique critical point, which provides a global minimum. We assign this point to $Z$ as its covariant $z(Z)$, thus solving our problem.

\section{Basics}

In all of the paper, we fix $n \geq 0$.

We consider the group $G=\operatorname{SL}(n+1, \mathbb{C})$ and its natural action on forms (homogeneous polynomials) in $n+1$ variables $X_{0}, \ldots, X_{n}$ by linear substitutions; this action will be on the right:

$$
F\left(X_{0}, X_{1}, \ldots, X_{n}\right) \cdot\left(a_{i j}\right)_{0 \leq i, j \leq n}=F\left(\sum_{j=0}^{n} a_{0 j} X_{j}, \ldots, \sum_{j=0}^{n} a_{n j} X_{j}\right) .
$$

The same action is used for Hermitian forms in $X_{0}, \ldots, X_{n}$. A Hermitian form can be considered as a bihomogeneous polynomial of bidegree $(1,1)$ in two sets of variables $X_{0}, \ldots, X_{n}$ and $\bar{X}_{0}, \ldots, \bar{X}_{n}$, where the action on the second set is through the complex conjugate of the matrix. The form $Q$ is Hermitian if $Q(\bar{X} ; X)=\bar{Q}(X ; \bar{X})$, where $\bar{Q}$ denotes the form obtained from $Q$ by replacing the coefficients with their complex conjugates. Hermitian forms can also be identified with Hermitian matrices, i.e., matrices $A$ such that $A^{\top}=\bar{A}$, where $A$ corresponds to $Q$ if $Q(\boldsymbol{x})=\overline{\boldsymbol{x}} A \boldsymbol{x}^{\top}$; then the action of $G$ is given by $A \cdot \gamma=\bar{\gamma}^{\top} A \gamma$. 
The group $G$ also acts on coordinates $\left(\xi_{0}, \ldots, \xi_{n}\right)$ on the right via the contragredient representation,

$$
\left(\xi_{0}, \ldots, \xi_{n}\right) \cdot \gamma=\left(\xi_{0}, \ldots, \xi_{n}\right) \gamma^{-\top}
$$

These actions are compatible in the sense that

$$
(Q \cdot \gamma)(\boldsymbol{x} \cdot \gamma)=Q(\boldsymbol{x})
$$

for Hermitian forms $Q$ and coordinate vectors $\boldsymbol{x}$.

\section{Point clusters}

The actions described above induce actions of $\operatorname{PSL}(n+1, \mathbb{C})=\operatorname{PGL}(n+1, \mathbb{C})$ on projective schemes over $\mathbb{C}$ and points in projective space $\mathbb{P}^{n}(\mathbb{C})$. The first specialises and the second generalises to an action on positive zero-cycles.

Definition 1. A positive zero-cycle or point cluster is a formal sum $Z=\sum_{j=1}^{m} P_{j}$ of points $P_{j} \in \mathbb{P}^{n}$. The number $m$ of points is the degree of $Z$, written $\operatorname{deg} Z$. If $L \subset \mathbb{P}^{n}$ is a linear subspace, we let $\left.Z\right|_{L}$ be the sum of those points in $Z$ that lie in $L$.

Definition 2. Let $Z$ be a point cluster in $\mathbb{P}^{n}$.

(1) $Z$ is split if there are two disjoint and nonempty linear subspaces $L_{1}, L_{2}$ of $\mathbb{P}^{n}$ such that $Z=\left.Z\right|_{L_{1}}+\left.Z\right|_{L_{2}}$. Otherwise, $Z$ is non-split.

(2) $Z$ is semi-stable if for every linear subspace $L \subset \mathbb{P}^{n}$, we have

$$
\left.(n+1) \operatorname{deg} Z\right|_{L} \leq(\operatorname{dim} L+1) \operatorname{deg} Z .
$$

(3) $Z$ is stable if for every linear subspace $\emptyset \neq L \subsetneq \mathbb{P}^{n}$, we have

$$
\left.(n+1) \operatorname{deg} Z\right|_{L}<(\operatorname{dim} L+1) \operatorname{deg} Z .
$$

Remark 3. Note that a split point cluster cannot be stable.

If we identify the cluster $Z=\sum_{j=1}^{m} P_{j}$, where $P_{j}=\left(a_{j 0}: a_{j 1}: \cdots: a_{j n}\right)$, with the form $F(Z)=\prod_{j=1}^{m}\left(a_{j 0} x_{0}+a_{j 1} x_{1}+\cdots+a_{j n} x_{n}\right)$ (up to scaling), then $Z$ is (semi-)stable if and only if $F(Z)$ is (semi-)stable in the sense of Geometric Invariant Theory; see [7].

If $n=1$, then the notions of stable and semi-stable defined here coincide with those defined in [8] (in Def. 4.1 and before Prop. 5.2) for binary forms.

Definition 4. Let $\mathcal{Z}_{m}$ denote the set of point clusters of degree $m$ in $\mathbb{P}^{n}(\mathbb{C}), \mathcal{Z}_{m}^{\text {sst }}$ the subset of semi-stable and $\mathcal{Z}_{m}^{\text {st }}$ the subset of stable point clusters. We denote by $Z_{m}(\mathbb{R})$ etc. the subset of point clusters fixed by complex conjugation, which acts via $\sum_{j} P_{j} \mapsto \sum_{j} \bar{P}_{j}$ 
For notational convenience, for a point cluster $Z$ and $-1 \leq k \leq n$ we define $\varphi_{Z}(k)=\max \left\{\left.\operatorname{deg} Z\right|_{L}: L \subset \mathbb{P}^{n}\right.$ a $k$-dimensional linear subspace $\}$.

Then $Z$ is semi-stable if and only if $\varphi_{Z}(k) \leq \frac{k+1}{n+1} \operatorname{deg} Z$ for all $0 \leq k \leq n$, and $Z$ is stable if and only if the inequality is strict for $0 \leq k<n$.

We let $\left\langle P, P^{\prime}\right\rangle=\bar{P}\left(P^{\prime}\right)^{\top}$ denote the standard Hermitian inner product on row vectors and $\|P\|^{2}=\langle P, P\rangle$ the corresponding norm. The next lemma is the basis for most of what follows.

Lemma 5. Let $Z \in Z_{m}$. Fix row vectors $P_{j}, j \in\{1, \ldots, m\}$, representing the points in $Z$, such that $\left\|P_{j}\right\|^{2}=1$. Then there is a constant $c>0$ such that for every positive definite Hermitian matrix $Q$ with eigenvalues $0<\lambda_{0} \leq \lambda_{1} \leq \cdots \leq \lambda_{n}$, we have

$$
\prod_{j=1}^{m}\left(\bar{P}_{j} Q P_{j}^{\top}\right) \geq c \prod_{k=0}^{n} \lambda_{k}^{\varphi_{Z}(k)-\varphi_{Z}(k-1)} .
$$

Proof. Let $B=b_{0}, \ldots, b_{n}$ be a unitary basis of $\mathbb{C}^{n+1}$. Let $E_{k}=\left\langle b_{0}, \ldots, b_{k}\right\rangle$ the subspace generated by the first $k+1$ basis vectors. By definition of $\varphi_{Z}$, the set $\Sigma(B) \subset S_{m}$ of permutations $\sigma$ with the following property is nonempty:

$$
P_{\sigma(j)} \notin E_{k} \quad \text { if } j>\varphi_{Z}(k) \text {. }
$$

Define $k_{\sigma}(j)=\min \left\{k: \sigma(j) \leq \varphi_{Z}(k)\right\}$; then $P_{\sigma(j)} \notin E_{k_{\sigma}(j)-1}$ if $\sigma \in \Sigma(B)$. Write $P_{j}=\sum_{i=0}^{n} \xi_{j i} b_{i}$ and define

$$
f_{\sigma}(B)=\prod_{j=1}^{m}\left(\sum_{i=k_{\sigma}(j)}^{n}\left|\xi_{\sigma(j), i}\right|^{2}\right)=\prod_{j=1}^{m}\left(\sum_{i=k_{\sigma}(j)}^{n}\left|\left\langle P_{\sigma(j)}, b_{i}\right\rangle\right|^{2}\right)
$$

and

$$
f(B)=\max \left\{f_{\sigma}(B): \sigma \in S_{m}\right\} .
$$

It is clear that $f_{\sigma}$ is continuous on the set of unitary bases and that $f_{\sigma}(B)>0$ if $\sigma \in \Sigma(B)$. This implies that $f$ is continuous and positive. Since the set of all unitary bases (i.e., $\mathrm{U}(n+1))$ is compact, there is some $c>0$ such that $f(B) \geq c$ for all $B$.

Now let $Q$ be a positive definite Hermitian matrix as in the statement of the Lemma. Let $B=b_{0}, \ldots, b_{n}$ be a unitary basis of eigenvectors of $Q$ such that $b_{j} Q=\lambda_{j} b_{j}$. We then have for $\sigma \in S_{m}$ and using notation introduced above

$$
\begin{aligned}
\prod_{j=1}^{m}\left(\bar{P}_{j} Q P_{j}^{\top}\right) & =\prod_{j=1}^{m}\left(\bar{P}_{\sigma(j)} Q P_{\sigma(j)}^{\top}\right)=\prod_{j=1}^{m}\left(\sum_{i=0}^{n} \lambda_{i}\left|\xi_{\sigma(j), i}\right|^{2}\right) \\
& \geq \prod_{j=1}^{m}\left(\lambda_{k_{\sigma}(j)} \sum_{i=k_{\sigma}(j)}^{n}\left|\xi_{\sigma(j), i}\right|^{2}\right) \\
& =f_{\sigma}(B) \prod_{j=1}^{m} \lambda_{k_{\sigma}(j)}=f_{\sigma}(B) \prod_{k=0}^{n} \lambda_{k}^{\varphi_{Z}(k)-\varphi_{Z}(k-1)}
\end{aligned}
$$


Taking the maximum over all $\sigma \in S_{m}$ now shows that

$$
\prod_{j=1}^{m}\left(\bar{P}_{j} Q P_{j}^{\top}\right) \geq f(B) \prod_{k=0}^{n} \lambda_{k}^{\varphi_{Z}(k)-\varphi_{Z}(k-1)} \geq c \prod_{k=0}^{n} \lambda_{k}^{\varphi_{Z}(k)-\varphi_{Z}(k-1)} .
$$

\section{The covariant}

Definition 6. Let $\widetilde{Z}_{m}$ denote the set of point clusters of degree $m$ with a choice of coordinates for the points, up to scaling the coordinates of the points with factors whose product is 1 . We will call $\widetilde{Z} \in \widetilde{Z}_{m}$ a point cluster with scaling. We define $\widetilde{Z}_{m}^{\text {st }}$ and $\widetilde{Z}_{m}^{\text {sst }}$ analogously.

For $\lambda \in \mathbb{C}^{\times}$and $\widetilde{Z} \in \widetilde{Z}_{m}$, we write $\lambda \widetilde{Z}$ for the cluster with scaling that we obtain by scaling one of the points in $\widetilde{Z}$ by $\lambda$. This defines an action of $\mathbb{C}^{\times}$on $\widetilde{Z}_{m}$ such that the quotient $\mathbb{C}^{\times} \backslash \widetilde{\mathcal{Z}}_{m}$ is $\mathcal{Z}_{m}$. If $\widetilde{Z} \in \tilde{\mathcal{Z}}_{m}$, then we write $Z$ for the image of $\widetilde{Z}$ in $\mathcal{Z}_{m}$.

Definition 7. For a point cluster with scaling $\widetilde{Z} \in \widetilde{Z}_{m}$, pick a representative $\sum_{j=1}^{m} P_{j}$ with row vectors $P_{j}$. Then, for $Q \in \mathscr{H}_{n, \mathbb{C}}$, represented by a Hermitian matrix, we define

$$
D_{\widetilde{Z}}(Q)=D(\tilde{Z}, Q)=\sum_{j=1}^{m} \log \left(\bar{P}_{j} Q P_{j}^{\top}\right)-\frac{m}{n+1} \log \operatorname{det} Q .
$$

$D(\tilde{Z}, Q)$ is clearly invariant under scaling of $Q$, and it does not depend on the choice of representative for $\widetilde{Z}$. Note also that for $\gamma \in G$,

$$
D(\tilde{Z} \cdot \gamma, Q \cdot \gamma)=D(\tilde{Z}, Q) .
$$

Furthermore, we have $D(\overline{\widetilde{Z}}, \bar{Q})=D(\tilde{Z}, Q)$ and $D(\lambda \tilde{Z}, Q)=\log |\lambda|^{2}+D(\widetilde{Z}, Q)$.

This function generalises the distance function used in Prop. 5.3 of [8]. We will now proceed to show that for stable clusters, there is a unique form $Q \in \mathscr{H}_{n, \mathbb{C}}$ that minimises this distance.

To that end, we now identify $\mathscr{H}_{n, \mathbb{C}}$ with the set of positive definite Hermitian matrices of determinant 1 . This is a real $n(n+2)$-dimensional submanifold of the space of all complex $(n+1) \times(n+1)$-matrices. $\operatorname{SL}(n+1, \mathbb{C})$ acts transitively on this space, and the tangent space $T$ at the identity matrix $I$ consists of the Hermitian matrices of trace zero. We say that a twice continuously differentiable function on $\mathscr{H}_{n, \mathbb{C}}$ is convex if its second derivative is positive semidefinite, and strictly convex if its second derivative is positive definite. Then the usual conclusions on convex functions apply.

Lemma 8. Let $\tilde{Z} \in \widetilde{Z}_{m}$ be a point cluster with scaling.

(1) The function $D_{\widetilde{Z}}$ is convex. 
(2) If $Z$ is non-split, then $D_{\tilde{Z}}$ is strictly convex.

(3) If $Z$ is semi-stable, then $D_{\widetilde{Z}}$ is bounded from below.

(4) If $Z$ is stable, then the sets $\left\{Q \in \mathscr{H}_{n, \mathbb{C}}: D_{\widetilde{Z}}(Q) \leq B\right\}$ are compact for all $B \in \mathbb{R}$.

Proof. Since scaling $\tilde{Z}$ only changes $D_{\widetilde{Z}}$ by an additive constant, we can assume that $\widetilde{Z}=P_{1}+\cdots+P_{m}$ with row vectors $P_{j}$ satisfying $\left\|P_{j}\right\|^{2}=1$.

(1) Since $D_{\widetilde{Z}}(Q \cdot \gamma)=D_{\widetilde{Z} \cdot \gamma^{-1}}(Q)$, we can assume that $Q=I$. We compute the second derivative at $\lambda=0$ of $\lambda \mapsto f(\lambda)=D_{\widetilde{Z}}(\exp (\lambda A))$, where $A \neq 0$ is a Hermitian trace-zero matrix (i.e., $A \in T$ ). We have

$$
\begin{aligned}
D_{\widetilde{Z}}(\exp (\lambda A)) & =\sum_{j} \log \left(1+\bar{P}_{j} A P_{j}^{\top} \cdot \lambda+\bar{P}_{j} A^{2} P_{j}^{\top} \cdot \lambda^{2} / 2+\ldots\right) \\
& =\sum_{j}\left(\bar{P}_{j} A P_{j}^{\top} \cdot \lambda+\left(\bar{P}_{j} A^{2} P_{j}^{\top}-\left(\bar{P}_{j} A P_{j}^{\top}\right)^{2}\right) \cdot \lambda^{2} / 2+\ldots\right)
\end{aligned}
$$

The second derivative therefore is

$$
\sum_{j}\left(\bar{P}_{j} A^{2} P_{j}^{\top}-\left(\bar{P}_{j} A P_{j}^{\top}\right)^{2}\right)=\sum_{j}\left(\left\|P_{j} \bar{A}\right\|^{2}\left\|P_{j}\right\|^{2}-\left|\left\langle P_{j} \bar{A}, P_{j}\right\rangle\right|^{2}\right) \geq 0
$$

by the Cauchy-Schwarz inequality. This shows that the second derivative is positive semidefinite, whence the first claim.

(2) As in (1), it suffices to consider the case $Q=I$, since the condition for $Z$ to be non-split is invariant under the action of $\operatorname{SL}(n+1, \mathbb{C})$. The second derivative in (1) vanishes exactly when $P_{j}$ is an eigenvector of $A$, for all $j$. Since $Z$ is non-split, this is only possible if $A$ is a scalar matrix: the $P_{j}$ must all be in the same eigenspace, and their span is the whole space. But $A \neq 0$ has trace zero, so $A$ cannot be a scalar matrix. So the second derivative at $I$ must be positive definite.

(3) By Lemma 5, we find some $c>0$ such that for $Q \in \mathscr{H}_{n}$, with eigenvalues $\lambda_{0} \leq \cdots \leq \lambda_{n}$, we have

$$
\prod_{j=1}^{m}\left(\bar{P}_{j} Q P_{j}^{\top}\right) \geq c \prod_{k=0}^{n} \lambda_{k}^{\varphi_{Z}(k)-\varphi_{Z}(k-1)} .
$$

With $\varphi_{Z}(k) \leq(k+1) \frac{m}{n+1}$, we obtain

$$
\begin{aligned}
D_{\widetilde{Z}}(Q) & \geq \log c+\sum_{k=0}^{n}\left(\varphi_{Z}(k)-\varphi_{Z}(k-1)\right) \log \lambda_{k} \\
& =\log c+m \log \lambda_{n}-\sum_{k=1}^{n} \varphi_{Z}(k-1)\left(\log \lambda_{k}-\log \lambda_{k-1}\right)
\end{aligned}
$$




$$
\begin{aligned}
& \geq \log c+m \log \lambda_{n}-\frac{m}{n+1} \sum_{k=1}^{n} k\left(\log \lambda_{k}-\log \lambda_{k-1}\right) \\
& =\log c+\frac{m}{n+1} \sum_{k=0}^{n} \log \lambda_{k} \\
& =\log c
\end{aligned}
$$

(recall that $\sum_{k} \log \lambda_{k}=\log \operatorname{det} Q=0$ ).

(4) We now use that $\varphi_{Z}(k) \leq(k+1) \frac{m}{n+1}-\frac{1}{n+1}$ for $0 \leq k \leq n-1$. The computation in the proof of (3) above then yields

$$
\begin{aligned}
D_{\widetilde{Z}}(Q) \geq \log c+m \log \lambda_{n}-\sum_{k=1}^{n} \varphi_{Z}(k-1)\left(\log \lambda_{k}-\log \lambda_{k-1}\right) \\
\geq \log c+m \log \lambda_{n} \\
\quad-\frac{m}{n+1} \sum_{k=1}^{n} k\left(\log \lambda_{k}-\log \lambda_{k-1}\right)+\frac{1}{n+1} \sum_{k=1}^{n}\left(\log \lambda_{k}-\log \lambda_{k-1}\right) \\
=\log c+\frac{1}{n+1}\left(\log \lambda_{n}-\log \lambda_{0}\right) .
\end{aligned}
$$

So $D_{\widetilde{Z}}(Q) \leq B$ implies that $\lambda_{n} / \lambda_{0}$ is bounded, but this implies that the subset of $Q \in \mathscr{H}_{n, \mathbb{C}}$ satisfying $D_{\widetilde{Z}}(Q) \leq B$ is also bounded. Since it is obviously closed, it must be compact.

Remark 9. Note that if $Z$ is not stable, then there are sets $\left\{Q: D_{\widetilde{Z}}(Q) \leq B\right\}$ that are not compact. Indeed, there is a linear subspace $L_{0} \subset \mathbb{C}^{n+1}$ of some dimension $0<k+1<n+1$ containing at least $(k+1) m /(n+1)$ points of $Z$. Let $L_{1}$ be its orthogonal complement. Let $Q_{\lambda}$ be the Hermitian matrix with eigenvalue $\lambda^{-(n-k)}$ on $L_{0}$ and eigenvalue $\lambda^{k+1}$ on $L_{1}$. Then we have for $\lambda \geq 1$ that

$D_{\widetilde{Z}}\left(Q_{\lambda}\right) \leq$ const. $+(k+1) \frac{m}{n+1} \log \lambda^{-(n-k)}+(n-k) \frac{m}{n+1} \log \lambda^{k+1}=$ const. ;

but the set $\left\{Q_{\lambda}: \lambda \geq 1\right\}$ is not relatively compact.

We also see that $D_{\widetilde{Z}}$ is not bounded from below when $Z$ is not semi-stable, since using the corresponding strict inequality, we find with a similar argument that

$$
D_{\widetilde{Z}}\left(Q_{\lambda}\right) \leq \text { const. }-\varepsilon \log \lambda
$$

for some $\varepsilon>0$.

Corollary 10. If $\widetilde{Z} \in \widetilde{Z}_{m}^{\text {st }}$, then the function $D_{\widetilde{Z}}$ has a unique critical point $z(Z)$ on $\mathscr{H}_{n, \mathbb{C}}$, and at this point $D_{\widetilde{Z}}$ achieves its global minimum $\log \theta(\widetilde{Z})$ (for some $\left.\theta(\widetilde{Z}) \in \mathbb{R}_{>0}\right)$. 
Proof. By Lemma 8 , we know that $D_{\widetilde{Z}}$ is strictly convex and also that for all $B$ the set $\left\{Q \in \mathscr{H}_{n, \mathbb{C}}: D_{\widetilde{Z}}(Q) \leq B\right\}$ is compact. The first property implies that every critical point must be a local minimum. By the second property, there exists a global minimum. If there were two distinct local minima, then on a path joining the two, there would have to be a local maximum, but then the second derivative would not be positive definite in this point, a contradiction. Hence there is a unique local minimum, which must then also be the global minimum and the unique critical point.

Since $D_{\lambda \tilde{Z}}=\log |\lambda|^{2}+D_{\tilde{Z}}$, the minimising point in $\mathscr{H}_{n, \mathbb{C}}$ does not depend on the scaling, so it only depends on $Z$, and the notation $z(Z)$ is justified.

Note that we have $\theta(\lambda \tilde{Z})=|\lambda|^{2} \theta(\tilde{Z})$.

Corollary 10 defines $z: \mathcal{Z}_{m}^{\text {st }} \rightarrow \mathscr{H}_{n, \mathbb{C}}$ and $\theta: \widetilde{Z}_{m}^{\text {st }} \rightarrow \mathbb{R}_{>0}$. The latter extends to

$$
\theta: \widetilde{Z}_{m} \longrightarrow \mathbb{R}_{\geq 0}
$$

with the definition $\theta(\tilde{Z})=\inf _{Q \in \mathscr{H}_{n, \mathbb{C}}} \exp (D(\widetilde{Z}, Q))$. By Lemma 8(3) we have $\theta(\widetilde{Z})>0$ if $\widetilde{Z} \in \tilde{Z}_{m}^{\text {sst }}$, and by the preceding remark, $\theta(\tilde{Z})=0$ if $\tilde{Z}$ is not semistable.

Corollary 11. The function $z: Z_{m}^{\text {st }} \rightarrow \mathscr{H}_{n, \mathbb{C}}$ is $\mathrm{SL}(n+1, \mathbb{C})$-equivariant. It also satisfies $z(\bar{Z})=\overline{z(Z)}$. In particular, z restricts to $z: Z_{m}^{\text {st }}(\mathbb{R}) \rightarrow \mathscr{H}_{n, \mathbb{R}}$.

The function $\theta: \widetilde{Z}_{m} \rightarrow \mathbb{R}_{\geq 0}$ is invariant under $\operatorname{SL}(n+1, \mathbb{C})$ and under complex conjugation.

Proof. The first statement follows from the invariance of $D$ (under the action of both $\operatorname{SL}(n+1, \mathbb{C})$ and complex conjugation) and the uniqueness of $z(Z)$. The second statement follows from the invariance of $D$.

Remark 12. In some cases the point $z(Z)$ is uniquely determined by symmetry considerations. Namely if the point cluster $Z \in Z_{m}^{\text {st }}$ is stabilised by a subgroup of $\operatorname{SL}(n+1, \mathbb{C})$ that fixes a unique point in $\mathscr{H}_{n, \mathbb{C}}$, then $z(Z)$ must be this point. See Lemma 3.1 in [8] for a precise statement. This observation facilitates the numerical computation of $z(Z)$, since it eliminates the need for finding numerically the minimum of the distance function on $\mathscr{H}_{n, \mathbb{C}}$.

Example 13. Consider a sum $Z$ of $n+2$ points in general position in $\mathbb{P}^{n}(\mathbb{C})$. Then $Z$ is stable. Since $\operatorname{PGL}(n+1, \mathbb{C})$ acts transitively on $(n+2)$-tuples of points in general position, we can assume that the points in $Z$ are the coordinate points together with the point $(1: \cdots: 1)$. Let this specific cluster be $Z_{0}$. The stabiliser of $Z_{0}$ in PGL $(n+1)$ is isomorphic to the symmetric group $S_{n+2}$; its preimage $\Gamma$ in $\operatorname{SL}(n+1, \mathbb{C})$ acts irreducibly on $\mathbb{C}^{n+1}$. By Schur's lemma, there is a unique (up to scaling) $\Gamma$-invariant positive definite Hermitian form. It can be checked that

$$
Q_{0}\left(x_{0}, \ldots, x_{n}\right)=\sum_{i=0}^{n}\left|x_{i}\right|^{2}+\sum_{0 \leq i<j \leq n}\left|x_{i}-x_{j}\right|^{2}=(n+2) \sum_{i=0}^{n}\left|x_{i}\right|^{2}-\left|\sum_{i=0}^{n} x_{i}\right|^{2}
$$


is invariant under $\Gamma$, hence $z\left(Z_{0}\right)=Q_{0}$. In general, we just have to find a matrix $\gamma$ such that $Z_{0} \cdot \gamma^{-\top}=Z$; then

$$
z(Z)=z\left(Z_{0} \cdot \gamma^{-\top}\right)=Q_{0} \cdot \gamma^{-\top} .
$$

Note that $Z_{0} \cdot \gamma^{-\top}=\sum_{j} P_{0, j} \gamma$ if $Z_{0}=\sum_{j} P_{0, j}$ and we think of the $P_{0, j}$ as row vectors. So if $Z=\sum_{j} P_{j}$, then the rows of $\gamma$ are coordinate vectors for the first $n+1$ points in $Z$, scaled in such a way that their sum is a coordinate vector for the last point.

\section{Reduction of point clusters}

We can now define when a point cluster is reduced.

Definition 14. Let $Z \in Z_{m}^{\text {st }}(\mathbb{R})$. We say that $Z$ is $L L L$-reduced, resp., Minkowskireduced if the positive definite real quadratic form corresponding to $z(Z)$ is LLLreduced, resp., Minkowski-reduced.

By definition, there is an essentially unique Minkowski-reduced representative in the $\operatorname{SL}(n+1, \mathbb{Z})$-orbit of a given point cluster $Z \in Z_{m}^{\text {st }}(\mathbb{R})$. On the other hand, for computational purposes, it is usually more convenient to work with LLL-reduced representatives. In order to find an LLL-reduced representative of $Z$ 's orbit, we compute the covariant $Q=z(Z)$. Then we use the LLL algorithm [6] to find $\gamma \in \operatorname{SL}(n+1, \mathbb{Z})$ such that $Q \cdot \gamma$ is LLL-reduced. Then $Z \cdot \gamma$ is an LLL-reduced representative of the orbit of $Z$.

Example 15. We can use our results to reduce pencils of quadrics in three variables whose generic member is smooth. These correspond to four points in general position in $\mathbb{P}^{2}$. We illustrate the method with a concrete example. Let

$$
\begin{aligned}
Q_{1}(x, y, z)= & 857211194051 x^{2}-10879213981695 x y-1296007209476 x z \\
& +34518126244996 y^{2}+8224075847095 y z+489854396055 z^{2}, \\
Q_{2}(x, y, z)= & 2274418654562 x^{2}-28865567091425 x y-3438665984061 x z \\
& +91586146842213 y^{2}+21820750429746 y z+1299719350945 z^{2}
\end{aligned}
$$

be a pair of quadrics. We first determine a good basis of the pencil spanned by $Q_{1}$ and $Q_{2}$ by reducing the binary cubic

$$
\operatorname{det}\left(x M_{1}+y M_{2}\right)=27348 x^{3}+215720 x^{2} y+567184 x y^{2}+497080 y^{3}
$$

with the approach described in [8]. Here $M_{1}$ and $M_{2}$ are the matrices of second partial derivatives of $Q_{1}$ and $Q_{2}$, respectively. This suggests the new basis

$$
Q_{1}^{\prime}=-21 Q_{1}+8 Q_{2}, \quad Q_{2}^{\prime}=-8 Q_{1}+3 Q_{2}
$$


with already somewhat smaller coefficients; the new binary cubic is

$$
-4 x^{3}+88 x^{2} y+112 x y^{2}-24 y^{3} .
$$

Now we find the four points of intersection numerically. We obtain

$$
\begin{aligned}
& P_{1}=(0.3038054131+0.0003625989 i:-0.0712511408+0.0000571409 i: 1), \\
& P_{2}=(0.3038054131-0.0003625989 i:-0.0712511408-0.0000571409 i: 1), \\
& P_{3}=(0.3038639670+0.0003672580 i:-0.0712419135+0.0000578751 i: 1), \\
& P_{4}=(0.3038639670-0.0003672580 i:-0.0712419135-0.0000578751 i: 1),
\end{aligned}
$$

and from this a matrix $\gamma \in \operatorname{SL}(3, \mathbb{C})$ that brings these points in standard position:

$$
\gamma^{-1}=\left(\begin{array}{rrr}
-13584.01-1762.69 i & 3186.66+407.04 i & -44719.72-5748.66 i \\
8318.54+10882.75 i & -1945.84-2556.21 i & 27338.35+35854.08 i \\
14176.55+2104.80 i & -3324.73-486.76 i & 46662.58+6870.37 i
\end{array}\right) .
$$

From this, we obtain a matrix representing $z\left(P_{1}+P_{2}+P_{3}+P_{4}\right)$ as

$$
\bar{\gamma}\left(\begin{array}{rrr}
3 & -1 & -1 \\
-1 & 3 & -1 \\
-1 & -1 & 3
\end{array}\right) \gamma^{\top}=\left(\begin{array}{rrrr}
241474533625.0 & -1532325529959.9 & -182541212588.9 \\
-1532325529959.9 & 9723681808257.5 & 1158352212636.4 \\
-182541212588.9 & 1158352212636.4 & 137990925143.2
\end{array}\right)
$$

(For the actual computation, more precision is needed than indicated by the numbers above.) An LLL computation applied to this Gram matrix suggests the transformation given by

$$
g=\left(\begin{array}{rrr}
3780 & 19276 & -12561 \\
-889 & -4515 & 2953 \\
12463 & 63400 & -41405
\end{array}\right)
$$

and indeed, if we apply the corresponding substitution to $Q_{1}^{\prime}$ and $Q_{2}^{\prime}$, we obtain the nice and small quadrics

$$
2 x^{2}-x y+x z+2 z^{2} \text { and }-2 x z+3 y^{2}-y z+2 z^{2} .
$$

\section{Reduction of ternary forms}

In this section, we apply the reduction theory of point clusters to ternary forms. The idea is to associate to a ternary form, or rather, to the plane curve it defines, a stable point cluster in a covariant way. This should be a purely geometric construction working over any base field of characteristic zero. 
We will only consider irreducible ternary forms $F$ of degree $d$. Assume that the curve defined by $F$ has $r$ nodes and no other singularities; then its genus is

$$
g=\frac{1}{2}(d-1)(d-2)-r,
$$

and by [2], Exercise IV.4.6, p. 337, the number of inflection points is

$$
6(g-1)+3 d=3 d(d-2)-6 r .
$$

We let $Z(F)$ be the sum of the inflection points, counted with multiplicity. When is $Z(F)$ stable? The first condition is that the multiplicity of any point must be less than $d(d-2)-2 r$. Now the multiplicity is 2 less than the order of tangency of the inflectional tangent, so it is at most $d-2$. Hence the condition is satisfied if $d-2<d(d-2)-2 r$, i.e., if $0<(d-1)(d-2) / 2-r=g$. The second condition is that the multiplicities of points on a line add up to less than $2 d(d-2)-4 r$. Since there are at most $d$ points on the curve on a line, this sum is at most $d(d-2)$. Hence the condition is satisfied if $r<d(d-2) / 4$.

In any case, if $F$ defines a nonsingular plane curve of positive genus, then $Z(F)$ is stable, and we can set $z(F)=z(Z(F))$. We then define $F$ to be reduced if $z(F)$ is reduced (i.e., if $Z(F)$ is reduced).

Example 16. If $F$ is a nonsingular cubic, then it defines a smooth curve $C$ of genus 1 , with Jacobian elliptic curve $E$. The 3-torsion subgroup $E[3]$ acts on $C$ by linear automorphisms of the ambient $\mathbb{P}^{2}$. The preimage of $E[3]$ in $\operatorname{SL}(3, \mathbb{C})$ is a nonabelian group $\Gamma$ of order 27 that acts irreducibly on $\mathbb{C}^{3}$. Therefore there is a unique $Q \in \mathscr{H}_{2, \mathbb{C}}$ that is invariant under the action of $E[3]$. This $Q$ is then $z(F)$. If we know explicit matrices $M_{T} \in \mathrm{SL}(3, \mathbb{C})$ for $T \in E[3]$ that give the action of $E[3]$ on $\mathbb{P}^{2}$, then we can compute a representative of $Q$ as a Hermitian matrix as

$$
Q=\sum_{T \in E[3]}{\overline{M_{T}}}^{\top} M_{T}
$$

compare [1], §6.

We get the same result if we consider the cluster of inflection points on $C$, since this cluster (which is a principal homogeneous space for the action of $E$ [3]) is invariant under the same group $\Gamma$. Numerically, however, the method using the action of $E[3]$ seems to be more stable. See [1], §6, for some more discussion and details.

In general, we have to find the inflection points numerically and then find the minimum of $D_{\tilde{Z}}$, also numerically. This can be done by a steepest descent method. We will illustrate this by reducing a ternary quartic. 
Example 17. Let

$$
\begin{array}{rl}
F(x, y, z)=3 & 90908548757 x^{4}-1083699236751 x^{3} y+835578482044 x^{3} z \\
& +1126610184312 x^{2} y^{2}-1737329379412 x^{2} y z \\
& +669777678687 x^{2} z^{2}-520542386163 x y^{3} \\
& +1204081445939 x y^{2} z-928398396271 x y z^{2} \\
& +238611653627 x z^{3}+90192376558 y^{4}-278168756247 y^{3} z \\
& +321720059816 y^{2} z^{2}-165373310794 y z^{3}+31877479532 z^{4} .
\end{array}
$$

We compute the inflection points as the intersection points of $F=0$ and $H=0$, where $H$ is the Hessian of $F$. This gives 24 coordinate vectors and defines the point cluster $\widetilde{Z}$. We then use a steepest descent method to find (an approximation to) $z(Z)$, represented by the matrix

$$
\left(\begin{array}{rrr}
367751.9942 & -254909.8720 & 196557.1210 \\
-254909.8720 & 176692.9800 & -136245.3974 \\
196557.1210 & -136245.3974 & 105056.8935
\end{array}\right) .
$$

LLL applied to this Gram matrix suggests the transformation

$$
\left(\begin{array}{rrr}
-7 & 23 & -89 \\
-34 & 118 & -443 \\
-31 & 110 & -408
\end{array}\right)
$$

which turns $F$ into

$3 x^{4}-3 x^{3} y+3 x^{3} z+x^{2} y^{2}-2 x^{2} z^{2}+x y^{2} z-x y z^{2}-2 x z^{3}+3 y^{4}-3 y^{3} z+y^{2} z^{2}-3 z^{4}$.

\section{References}

[1] J. E. Cremona, T. A. Fisher, and M. Stoll, Minimisation and reduction of 2-, 3- and 4coverings of elliptic curves. Algebra Number Theory 4 (2010), 763-820. Zbl 05809198 MR 2728489

[2] R. Hartshorne, Algebraic geometry. Grad. Texts in Math. 52, Springer-Verlag, New York 1977. Zbl 0367.14001 MR 0463157

[3] C. Hermite, Note sur la réduction des fonctions homogènes à coefficients entiers et à deux indéterminées. J. Reine Angew. Math. 36 (1848), 357-364; also in Euvres de Charles Hermite, publiés par Émile Picard, Tome I, Gauthier-Villars, Paris 1905, 84-93.

[4] C. Hermite, Sur l'introduction des variables continues dans la théorie des nombres. $J$. Reine Angew. Math. 41 (1850), 191-216; also in: CEuvres de Charles Hermite, publiés par Émile Picard, Tome I, Gauthier-Villars, Paris 1905, 164-192, Sections V and VI. 
[5] G. Julia, Étude sur les formes binaires non quadratiques à indéterminées réelles ou complexes, ou à indéterminées conjugées. Mém. Acad. Sci. Inst. France (2) 55 (1917), 1-296; also in Euvres de Gaston Julia, Vol. V, Gauthier-Villars, Paris 1970, 51-342.

[6] A. K. Lenstra, H. W. Lenstra, Jr., and L. Lovász, Factoring polynomials with rational coefficients. Math. Ann. 261 (1982), 515-534. Zbl 0488.12001 MR 682664

[7] D. Mumford, J. Fogarty, and F. Kirwan, Geometric invariant theory. Ergeb. Math. Grenzgeb. (2) 34, 3rd ed., Springer-Verlag, Berlin 1994. Zbl 0797.14004 MR 1304906

[8] M. Stoll and J. E. Cremona, On the reduction theory of binary forms. J. Reine Angew. Math. 565 (2003), 79-99. Zbl 1153.11317 MR 2024647

Received August 31, 2009; revised March 8, 2010

M. Stoll, Mathematisches Institut, Universität Bayreuth, Universitätsstr. 30, 95447 Bayreuth, Germany

E-mail: Michael.Stoll@uni-bayreuth.de 